\title{
Variational approach to a class of $p$-Laplacian systems on time scales
}

Jianwen Zhou and Yongkun Li*

${ }^{\text {*Correspondence: yklie@ynu.edu.cn }}$
Department of Mathematics,
Yunnan University, Kunming,
Yunnan 650091, People's Republic
of China

"Correspondence: yklie@ynu.edu.cn Yunnan University, Kunming Yunnan 650091, People's Republic of China

\begin{abstract}
In this paper, we present a recent approach via variational methods and critical point theory to obtain the existence of nontrivial periodic solutions for a class of $p$-Laplacian systems on time scales. By establishing a proper variational setting, three existence results are obtained. Finally, two examples are presented to illustrate the feasibility and effectiveness of our results.
\end{abstract}

MSC: $34 \mathrm{~N} 05 ; 37 \mathrm{~J} 45 ; 34 \mathrm{C} 25$

Keywords: variational approach; time scales; $p$-Laplacian systems; critical points

\section{Introduction}

Consider the $p$-Laplacian system on the time scale $\mathbb{T}$

$$
\left(\left|u^{\Delta}(t)\right|^{p-2} u^{\Delta}(t)\right)^{\Delta}=\nabla F(\sigma(t), u(\sigma(t))), \quad \Delta \text {-a.e. } t \in \mathbb{T}^{\kappa},
$$

where $p>1, u^{\Delta}(t)$ denotes the delta (or Hilger) derivative of $u$ at $t, u^{\Delta^{2}}(t)=\left(u^{\Delta}\right)^{\Delta}(t), \sigma$ is the forward jump operator, $T$ is a positive constant, $\mathbb{T}$ is $T$-periodic and $0, T \in \mathbb{T}, F$ : $\mathbb{T} \times \mathbb{R}^{N} \rightarrow \mathbb{R}$ satisfies the following assumption:

(A) $F(t, x)$ is $\Delta$-measurable and $T$-periodic in $t$ for every $x \in \mathbb{R}^{N}$ and continuously differentiable in $x$ for $t \in[0, T]_{\mathbb{T}}$, and there exist $a \in C\left(\mathbb{R}^{+}, \mathbb{R}^{+}\right)$,

$b^{\sigma} \in L_{\Delta}^{1}\left([0, T)_{\mathbb{T}}, \mathbb{R}^{+}\right)$such that

$$
|F(t, x)| \leq a(|x|) b(t), \quad|\nabla F(t, x)| \leq a(|x|) b(t)
$$

for all $x \in \mathbb{R}^{N}$ and $t \in[0, T]_{\mathbb{T}}$, where $\nabla F(t, x)$ denotes the gradient of $F(t, x)$ in $x$. Problem (1.1) covers the $p$-Laplacian system (for when $\mathbb{T}=\mathbb{R}$ )

$$
\left(\left|u^{\prime}(t)\right|^{p-2} u^{\prime}(t)\right)^{\prime}=\nabla F(t, u(t)), \quad \text { a.e. } t \in \mathbb{R},
$$

the second-order Hamiltonian system on time scale $\mathbb{T}$ (for when $p=2$ )

$$
u^{\Delta^{2}}(t)=\nabla F(\sigma(t), u(\sigma(t))), \quad \Delta \text {-a.e. } t \in \mathbb{T}^{\kappa}
$$

and the second-order Hamiltonian system (for when $p=2, \mathbb{T}=\mathbb{R}$ )

$$
\ddot{u}(t)=\nabla F(t, u(t)), \quad t \in \mathbb{R}
$$

\section{Springer}

O2013 Zhou and Li; licensee Springer. This is an Open Access article distributed under the terms of the Creative Commons Attribution License (http://creativecommons.org/licenses/by/2.0), which permits unrestricted use, distribution, and reproduction in any medium, provided the original work is properly cited. 
as well as the second-order discrete Hamiltonian system (for when $p=2, \mathbb{T}=\mathbb{Z}, T \geq 2$ )

$$
\Delta^{2} u(t)=\nabla F(t+1, u(t+1)), \quad t \in \mathbb{Z}
$$

In recent years, many authors have been devoted to the investigation concerning the existence of periodic solutions of (1.2) by using critical point theory (see [1, 2]). In [3], the authors studied Sobolev's spaces on time scales and their properties. As applications, they presented a recent approach via variational methods and the critical point theory to obtain the existence of solutions of (1.3). System (1.4) has also been investigated by several authors using various techniques and different conditions on the nonlinearities, such as the bounded or convex potential conditions (see [4]), the coercive type potential condition (see [5]), the even type potential condition (see [6]), the subquadratic potential condition in the Rabinowitz sense (see [7]) and the sublinear nonlinearity condition (see [8]). Moreover, many authors have paid much attention to study the periodic solutions of $(1.5)$ (see $[9,10])$. But, to the best of our knowledge, the existence of solutions for problems (1.1) have not been studied yet.

The study of dynamical systems on time scales is now an active area of research. One of the reasons for this is the fact that the study on time scales unifies the study of both discrete and continuous processes, besides many others. The pioneering works in this direction are Refs. [11-14]. The theory of time scales was initiated by Hilger in his Ph.D. thesis in 1988, providing a rich theory that unifies and extends discrete and continuous analysis $[15,16]$. A time scale $\mathbb{T}$ is an arbitrary nonempty closed subset of the real numbers, which has the topology inherited from the real numbers with the standard topology. The two most popular examples are $\mathbb{T}=\mathbb{R}$ and $\mathbb{T}=\mathbb{Z}$. The time scales calculus has a tremendous potential for applications in some mathematical models of real processes and phenomena studied in physics, chemical technology, population dynamics, biotechnology and economics, neural networks, social sciences (see [11]). For example, it can model insect populations that are continuous while in season (and may follow a difference scheme with variable stepsize), die out in winter, while their eggs are incubating or dormant, and then hatch in a new season, giving rise to a nonoverlapping population.

In recent years, dynamic equations on time scales have generated a considerable amount of interest and attracted many researchers (see [17-24]). There have been many approaches to study the existence and multiplicity of solutions for dynamic equations on time scales, such as the method of lower and upper solutions, fixed-point theory, coincidence degree theory and so on. In [17], authors used the fixed point theorem of strictset-contraction to study the existence of positive periodic solutions for functional dynamic equations with impulse effects on time scales. However, the study of the existence and multiplicity of solutions for dynamic equations on time scales using variational method has received considerably less attention (see, for example, [3, 20, 24]). Also, as is well known, it is very difficult to use Hilger's integral to deal with the existence of solutions to a dynamic equation on time scales since it is only concerned with antiderivatives. Therefore, it is still worth making an attempt to extend variational methods to study of periodic solutions for various Hamiltonian systems because there are tremendous applications on periodic solutions to discrete or continuous Hamiltonian systems theoretically and practically [25-32].

Our purpose of this paper is to study the variational structure of problem (1.1) in an appropriate space of functions and the existence of periodic solutions for problem (1.1) by some critical point theorems. 


\section{Preliminaries and statements}

In this section, we present some fundamental definitions and results from the calculus on time scales and Sobolev's spaces on time scales that will be required below. We first briefly recall some basic definitions and results concerning time scales.

Definition 2.1 ([15, Definition 1.1]) Let $\mathbb{T}$ be a time scale, for $t \in \mathbb{T}$, the forward jump operator $\sigma: \mathbb{T} \rightarrow \mathbb{T}$ is defined by

$$
\sigma(t)=\inf \{s \in \mathbb{T}, s>t\} \quad \text { for all } t \in \mathbb{T},
$$

while the backward jump operator $\rho: \mathbb{T} \rightarrow \mathbb{T}$ is defined by

$$
\rho(t)=\sup \{s \in \mathbb{T}, s<t\} \quad \text { for all } t \in \mathbb{T}
$$

(supplemented by $\inf \emptyset=\sup \mathbb{T}$ and $\sup \emptyset=\inf \mathbb{T}$, where $\emptyset$ denotes the empty set). A point $t \in \mathbb{T}$ is called right-scattered, left-scattered if $\sigma(t)>t, \rho(t)<t$ hold, respectively. Points that are right-scattered and left-scattered at the same time are called isolated. Also, if $t<$ $\sup \mathbb{T}$ and $\sigma(t)=t$, then $t$ is called right-dense, and if $t>\inf \mathbb{T}$ and $\rho(t)=t$, then $t$ is called left-dense. Points that are right-dense and left-dense at the same time are called dense. The set $\mathbb{T}^{\kappa}$ is derived from the time scale $\mathbb{T}$ as follows: If $\mathbb{T}$ has a left-scattered maximum $m$, then $\mathbb{T}^{\kappa}=\mathbb{T}-\{m\}$; otherwise, $\mathbb{T}^{\kappa}=\mathbb{T}$.

When $a, b \in \mathbb{T}, a<b$, we denote the intervals $[a, b]_{\mathbb{T}},[a, b)_{\mathbb{T}}$ and $(a, b]_{\mathbb{T}}$ in $\mathbb{T}$ by

$$
[a, b]_{\mathbb{T}}=[a, b] \cap \mathbb{T}, \quad[a, b)_{\mathbb{T}}=[a, b) \cap \mathbb{T}, \quad(a, b]_{\mathbb{T}}=(a, b] \cap \mathbb{T},
$$

respectively. Note that $[a, b]_{\mathbb{T}}^{\kappa}=[a, b]_{\mathbb{T}}$ if $b$ is left-dense and $[a, b]_{\mathbb{T}}^{\kappa}=[a, b)_{\mathbb{T}}=[a, \rho(b)]_{\mathbb{T}}$ if $b$ is left-scattered. We define $[a, b]_{\mathbb{T}}^{\kappa^{2}}=\left([a, b]_{\mathbb{T}}^{\kappa}\right)^{\kappa}$, therefore $[a, b]_{\mathbb{T}}^{\kappa^{2}}=[a, b]_{\mathbb{T}}$ if $b$ is left-dense and $[a, b]_{\mathbb{T}}^{\kappa^{2}}=[a, \rho(b)]_{\mathbb{T}}^{\kappa}$ if $b$ is left-scattered.

Definition 2.2 ([15, Definition 1.10]) Assume that $f: \mathbb{T} \rightarrow \mathbb{R}$ is a function, and let $t \in \mathbb{T}^{\kappa}$. Then we define $f^{\Delta}(t)$ to be the number (provided it exists) with the property that given any $\epsilon>0$, there is a neighborhood $U$ of $t$ (i.e., $U=(t-\delta, t+\delta) \cap \mathbb{T}$ for some $\delta>0$ ) such that

$$
\left|[f(\sigma(t))-f(s)]-f^{\Delta}(t)[\sigma(t)-s]\right| \leq \epsilon|\sigma(t)-s| \quad \text { for all } s \in U \text {. }
$$

We call $f^{\Delta}(t)$ the delta (or Hilger) derivative of $f$ at $t$. The function $f$ is delta (or Hilger) differentiable on $\mathbb{T}^{\kappa}$ provided $f^{\Delta}(t)$ exists for all $t \in \mathbb{T}^{\kappa}$. The function $f^{\Delta}: \mathbb{T}^{\kappa} \rightarrow \mathbb{R}$ is then called the delta derivative of $f$ on $\mathbb{T}^{\kappa}$.

Definition 2.3 ([3, Definition 2.3]) Assume that $f: \mathbb{T} \rightarrow \mathbb{R}^{N}$ is a function $f(t)=\left(f^{1}(t)\right.$, $\left.f^{2}(t), \ldots, f^{N}(t)\right)$, and let $t \in \mathbb{T}^{\kappa}$. Then we define $f^{\Delta}(t)=\left(f^{1^{\Delta}}(t), f^{2^{\Delta}}(t), \ldots, f^{N^{\Delta}}(t)\right)$ (provided it exists). We call $f^{\Delta}(t)$ the delta (or Hilger) derivative of $f$ at $t$. The function $f$ is delta (or Hilger) differentiable provided $f^{\Delta}(t)$ exists for all $t \in \mathbb{T}^{\kappa}$. The function $f^{\Delta}: \mathbb{T}^{\kappa} \rightarrow \mathbb{R}^{N}$ is then called the delta derivative of $f$ on $\mathbb{T}^{\kappa}$. 
Definition 2.4 ([15, Definition 2.7]) For a function $f: \mathbb{T} \rightarrow \mathbb{R}$, we shall talk about the second derivative $f^{\Delta^{2}}$ provided $f^{\Delta}$ is differentiable on $\mathbb{T}^{\kappa^{2}}=\left(\mathbb{T}^{\kappa}\right)^{\kappa}$ with the derivative $f^{\Delta^{2}}=$ $\left(f^{\Delta}\right)^{\Delta}: \mathbb{T}^{\kappa^{2}} \rightarrow \mathbb{R}$

Definition 2.5 ([3, Definition 2.5]) For a function $f: \mathbb{T} \rightarrow \mathbb{R}^{N}$, we shall talk about the second derivative $f^{\Delta^{2}}$ provided $f^{\Delta}$ is differentiable on $\mathbb{T}^{\kappa^{2}}=\left(\mathbb{T}^{\kappa}\right)^{\kappa}$ with the derivative $f^{\Delta^{2}}=$ $\left(f^{\Delta}\right)^{\Delta}: \mathbb{T}^{\kappa^{2}} \rightarrow \mathbb{R}^{N}$

The $\Delta$-measure $\mu_{\Delta}$ and $\Delta$-integration are defined as those in [32].

Definition 2.6 ([3, Definition 2.7]) Assume that $f: \mathbb{T} \rightarrow \mathbb{R}^{N}$ is a function, $f(t)=$ $\left(f^{1}(t), f^{2}(t), \ldots, f^{N}(t)\right)$, and let $A$ be a $\Delta$-measurable subset of $\mathbb{T}$. $f$ is integrable on $A$ if and only if $f^{i}(i=1,2, \ldots, N)$ are integrable on $A$, and $\int_{A} f(t) \Delta t=\left(\int_{A} f^{1}(t) \Delta t, \int_{A} f^{2}(t) \Delta t, \ldots\right.$, $\left.\int_{A} f^{N}(t) \Delta t\right)$.

Definition 2.7 ([33, Definition 2.3]) Let $B \subset \mathbb{T}$. $B$ is called a $\Delta$-null set if $\mu_{\Delta}(B)=0$. Say that a property $P$ holds $\Delta$-almost everywhere ( $\Delta$-a.e.) on $B$, or for $\Delta$-almost all $(\Delta$-a.a.) $t \in B$ if there is a $\Delta$-null set $E_{0} \subset B$ such that $P$ holds for all $t \in B \backslash E_{0}$.

For $p \in \mathbb{R}, p \geq 1$, we set the space

$$
L_{\Delta}^{p}\left([0, T)_{\mathbb{T}}, \mathbb{R}^{N}\right)=\left\{u:[0, T)_{\mathbb{T}} \rightarrow \mathbb{R}^{N}: \int_{[0, T)_{\mathbb{T}}}|f(t)|^{p} \Delta t<+\infty\right\}
$$

with the norm

$$
\|f\|_{L_{\Delta}^{p}}=\left(\int_{[0, T)_{\mathbb{T}}}|f(t)|^{p} \Delta t\right)^{\frac{1}{p}} .
$$

We have the following theorem.

Theorem 2.1 ([3, Theorem 2.1]) Let $p \in \mathbb{R}$ be such that $p \geq 1$. Then the space $L_{\Delta}^{p}\left([0, T)_{\mathbb{T}}\right.$, $\left.\mathbb{R}^{N}\right)$ is a Banach space together with the norm $\|\cdot\|_{L_{\Delta}^{p}}$. Moreover, $L_{\Delta}^{2}\left([a, b)_{\mathbb{T}}, \mathbb{R}^{N}\right)$ is a Hilbert space together with the inner product given for every $(f, g) \in L_{\Delta}^{p}\left([a, b)_{\mathbb{T}}, \mathbb{R}^{N}\right) \times$ $L_{\Delta}^{p}\left([a, b)_{\mathbb{T}}, \mathbb{R}^{N}\right)$ by

$$
\langle f, g\rangle_{L_{\Delta}^{2}}=\int_{[a, b)_{\mathbb{T}}}(f(t), g(t)) \Delta t
$$

where $(\cdot, \cdot)$ denotes the inner product in $\mathbb{R}^{N}$.

Definition 2.8 ([3, Definition 2.11]) A function $f:[a, b]_{\mathbb{T}} \rightarrow \mathbb{R}^{N}, f(t)=\left(f^{1}(t), f^{2}(t), \ldots\right.$, $\left.f^{N}(t)\right)$. We say that $f$ is absolutely continuous on $[a, b]_{\mathbb{T}}\left(\right.$ i.e., $\left.f \in A C\left([a, b]_{\mathbb{T}}, \mathbb{R}^{N}\right)\right)$ if for every $\epsilon>0$, there exists $\delta>0$ such that if $\left\{\left[a_{k}, b_{k}\right)_{\mathbb{T}}\right\}_{k=1}^{n}$ is a finite pairwise disjoint family of subintervals of $[a, b]_{\mathbb{T}}$ satisfying $\sum_{k=1}^{n}\left(b_{k}-a_{k}\right)<\delta$, then $\sum_{k=1}^{n}\left|f\left(b_{k}\right)-f\left(a_{k}\right)\right|<\epsilon$.

Now, we recall the Sobolev space $W_{\Delta, T}^{1, p}\left([0, T]_{\mathbb{T}}, \mathbb{R}^{N}\right)$ on $[0, T]_{\mathbb{T}}$ defined in [3]. For the sake of convenience, in the sequel, we let $u^{\sigma}(t)=u(\sigma(t))$. 
Definition 2.9 ([3, Definition 2.12]) Let $p \in \mathbb{R}$ be such that $p>1$ and $u:[0, T]_{\mathbb{T}} \rightarrow \mathbb{R}^{N}$. We say that $u \in W_{\Delta, T}^{1, p}\left([0, T]_{\mathbb{T}}, \mathbb{R}^{N}\right)$ if and only if $u \in L_{\Delta}^{p}\left([0, T)_{\mathbb{T}}, \mathbb{R}^{N}\right)$ and there exists $g$ : $[0, T]_{\mathbb{T}}^{\kappa} \rightarrow \mathbb{R}^{N}$ such that $g \in L_{\Delta}^{p}\left([0, T)_{\mathbb{T}}, \mathbb{R}^{N}\right)$ and

$$
\int_{[0, T)_{\mathbb{T}}}\left(u(t), \phi^{\Delta}(t)\right) \Delta t=-\int_{[0, T)_{\mathbb{T}}}\left(g(t), \phi^{\sigma}(t)\right) \Delta t, \quad \forall \phi \in C_{T, r d}^{1}\left([0, T]_{\mathbb{T}}, \mathbb{R}^{N}\right) .
$$

For $p \in \mathbb{R}, p>1$, we denote

$$
V_{\Delta, T}^{1, p}\left([0, T]_{\mathbb{T}}, \mathbb{R}^{N}\right)=\left\{x \in A C\left([0, T]_{\mathbb{T}}, \mathbb{R}^{N}\right): x^{\Delta} \in L_{\Delta}^{p}\left([0, T)_{\mathbb{T}}, \mathbb{R}^{N}\right), x(0)=x(T)\right\} .
$$

It follows from Remark 2.2 in [3] that

$$
V_{\Delta}^{1, p}\left([0, T]_{\mathbb{T}}, \mathbb{R}_{N}\right) \subset W_{\Delta}^{1, p}\left([0, T]_{\mathbb{T}}, \mathbb{R}^{N}\right)
$$

is true for every $p \in \mathbb{R}$ with $p>1$. These two sets are, as a class of functions, equivalent. It is the characterization of functions in $W_{\Delta, T}^{1, p}\left([0, T]_{\mathbb{T}}, \mathbb{R}^{N}\right)$ in terms of functions in $V_{\Delta, T}^{1, p}\left([0, T]_{\mathbb{T}}, \mathbb{R}^{N}\right)$ too. That is the following theorem.

Theorem 2.2 ([3, Theorem 2.5]) Suppose that $u \in W_{\Delta, T}^{1, p}\left([0, T]_{\mathbb{T}}, \mathbb{R}^{N}\right)$ for some $p \in \mathbb{R}$ with $p>1$, and that (2.1) holds for $g \in L_{\Delta}^{p}\left([0, T)_{\mathbb{T}}, \mathbb{R}^{N}\right)$. Then there exists a unique function $x \in V_{\Delta, T}^{1, p}\left([0, T]_{\mathbb{T}}, \mathbb{R}^{N}\right)$ such that the equalities

$$
x=u, \quad x^{\Delta}=g, \quad \Delta \text {-a.e. on }[0, T)_{\mathbb{T}}
$$

are satisfied and

$$
\int_{[0, T)_{\mathbb{T}}} g(t) \Delta t=0
$$

By identifying $u \in W_{\Delta, T}^{1, p}\left([0, T]_{\mathbb{T}}, \mathbb{R}^{N}\right)$ with its absolutely continuous representative $x \in$ $V_{\Delta, T}^{1, p}\left([0, T]_{\mathbb{T}}, \mathbb{R}^{N}\right)$ for which (2.2) holds, the set $W_{\Delta, T}^{1, p}\left([0, T]_{\mathbb{T}}, \mathbb{R}^{N}\right)$ can be endowed with the structure of a Banach space. That is, the following theorem.

Theorem 2.3 ([24, Theorem 2.21]) Assume $p \in \mathbb{R}$ and $p>1$. The set $W_{\Delta, T}^{1, p}\left([0, T]_{\mathbb{T}}, \mathbb{R}^{N}\right)$ is a Banach space together with the norm defined as

$$
\begin{aligned}
& \|u\|_{W_{\Delta, T}^{1, p}}=\left(\int_{[0, T)_{\mathbb{T}}}|u(t)|^{p} \Delta t+\int_{[0, T)_{\mathbb{T}}}\left|u^{\Delta}(t)\right|^{p} \Delta t\right)^{\frac{1}{p}}, \\
& \forall u \in W_{\Delta, T}^{1, p}\left([0, T]_{\mathbb{T}}, \mathbb{R}^{N}\right) .
\end{aligned}
$$

Moreover, the set $H_{\Delta, T}^{1}=W_{\Delta, T}^{1,2}\left([0, T]_{\mathbb{T}}, \mathbb{R}^{N}\right)$ is a Hilbert space together with the inner product

$$
\langle u, v\rangle_{H_{\Delta, T}^{1}}=\int_{[0, T)_{\mathbb{T}}}(u(t), v(t)) \Delta t+\int_{[0, T)_{\mathbb{T}}}\left(u^{\Delta}(t), v^{\Delta}(t)\right) \Delta t, \quad \forall u, v \in H_{\Delta, T}^{1} .
$$


Similar to the general Banach space $W_{T}^{1, p}, W_{\Delta, T}^{1, p}\left([0, T]_{\mathbb{T}}, \mathbb{R}^{N}\right)$ is a reflexive and uniformly convex Banach space.

The Banach space $W_{\Delta, T}^{1, p}\left([0, T]_{\mathbb{T}}, \mathbb{R}^{N}\right)$ has some important properties.

Theorem 2.4 ([24, Theorem 2.23]) There exists $K=K(p)>0$ such that the inequality $\|u\|_{\infty} \leq K\|u\|_{W_{\Delta, T}^{1, p}}$ holds for all $u \in W_{\Delta, T}^{1, p}\left([0, T]_{\mathbb{T}}, \mathbb{R}^{N}\right)$, where $\|u\|_{\infty}=\max _{t \in[0, T]_{\mathbb{T}}}|u(t)|$. Moreover, if $\int_{[0, T)_{\mathbb{T}}} u(t) \Delta t=0$, then $\|u\|_{\infty} \leq K\left\|u^{\Delta}\right\|_{L_{\Delta}^{p}}$.

Theorem 2.5 ([24, Theorem 2.25]) If the sequence $\left\{u_{k}\right\}_{k \in \mathbb{N}} \subset W_{\Delta, T}^{1, p}\left([0, T]_{\mathbb{T}}, \mathbb{R}^{N}\right)$ converges weakly to $u$ in $W_{\Delta, T}^{1, p}\left([0, T]_{\mathbb{T}}, \mathbb{R}^{N}\right)$, then $\left\{u_{k}\right\}_{k \in \mathbb{N}}$ converges strongly in $C\left([0, T]_{\mathbb{T}}, \mathbb{R}^{N}\right)$ to $u$.

Remark $2.1\left(\left[24\right.\right.$, Remark 2.26]) The immersion $W_{\Delta, T}^{1, p}\left([0, T]_{\mathbb{T}}, \mathbb{R}^{N}\right) \hookrightarrow C\left([0, T]_{\mathbb{T}}, \mathbb{R}^{N}\right)$ is compact.

Theorem 2.6 ([24, Theorem 2.27]) Let $L:[0, T]_{\mathbb{T}} \times \mathbb{R}^{N} \times \mathbb{R}^{N} \rightarrow \mathbb{R},(t, x, y) \rightarrow L(t, x, y)$ be Lebesgue $\Delta$-measurable in $t$ for each $(x, y) \in \mathbb{R}^{N} \times \mathbb{R}^{N}$ and continuously differentiable in $(x, y)$ for every $t \in[0, T]_{\mathbb{T}}$. If there exist $a \in C\left(\mathbb{R}^{+}, \mathbb{R}^{+}\right), b, c \in[0, T]_{\mathbb{T}} \rightarrow \mathbb{R}^{+}, b^{\sigma} \in$ $L_{\Delta}^{1}\left([0, T)_{\mathbb{T}}, \mathbb{R}^{+}\right)$and $c^{\sigma} \in L_{\Delta}^{q}\left([0, T)_{\mathbb{T}}, \mathbb{R}^{+}\right)(1<q<+\infty)$ such that for $\Delta$-almost $t \in[0, T]_{\mathbb{T}}$ and every $(x, y) \in \mathbb{R}^{N} \times \mathbb{R}^{N}$, one has

$$
\begin{aligned}
& |L(t, x, y)| \leq a(|x|)\left(b(t)+|y|^{p}\right), \\
& \left|L_{x}(t, x, y)\right| \leq a(|x|)\left(b(t)+|y|^{p}\right), \\
& \left|L_{y}(t, x, y)\right| \leq a(|x|)\left(c(t)+|y|^{p-1}\right),
\end{aligned}
$$

where $\frac{1}{p}+\frac{1}{q}=1$, then the functional $\Phi: W_{\Delta, T}^{1, p}\left([0, T]_{\mathbb{T}}, \mathbb{R}^{N}\right) \rightarrow \mathbb{R}$, defined as

$$
\Phi(u)=\int_{[0, T)_{\mathbb{T}}} L\left(\sigma(t), u^{\sigma}(t), u^{\Delta}(t)\right) \Delta t
$$

is continuously differentiable on $W_{\Delta, T}^{1, p}\left([0, T]_{\mathbb{T}}, \mathbb{R}^{N}\right)$ and

$$
\left\langle\Phi^{\prime}(u), v\right\rangle=\int_{[0, T)_{\mathbb{T}}}\left[\left(L_{x}\left(\sigma(t), u^{\sigma}(t), u^{\Delta}(t)\right), v^{\sigma}(t)\right)+\left(L_{y}\left(\sigma(t), u^{\sigma}(t), u^{\Delta}(t)\right), v^{\Delta}(t)\right)\right] \Delta t .
$$

To prove our main results, we need the following definitions and theorems.

Theorem 2.7 ([18, Lemma 2.7]) Let $\mathbb{T}$ be a $T$-periodic time scale, then $\sigma(t+T)=\sigma(t)+T$ for all $t \in \mathbb{T}$.

Definition $2.10\left(\left[4, \mathrm{P}_{81}\right]\right)$ Let $X$ be a real Banach space, $I \in C^{1}(X, \mathbb{R})$ and $c \in \mathbb{R}$. $I$ is said to be satisfying the (PS) $)_{c}$-condition on $X$ if the existence of a sequence $\left\{x_{n}\right\} \subseteq X$, such that $I\left(x_{n}\right) \rightarrow c$ and $I^{\prime}\left(x_{n}\right) \rightarrow 0$ as $n \rightarrow \infty$, implies that $c$ is a critical value of $I$.

Definition $2.11\left(\left[4, \mathrm{P}_{81}\right]\right)$ Let $X$ be a real Banach space and $I \in C^{1}(X, \mathbb{R}) . I$ is said to be satisfying the P.S. condition on $X$ if any sequence $\left\{x_{n}\right\} \subseteq X$, for which $I\left(x_{n}\right)$ is bounded and $I^{\prime}\left(x_{n}\right) \rightarrow 0$ as $n \rightarrow \infty$, possesses a convergent subsequence in $X$. 
Remark 2.2 It is clear that the P.S. condition implies the (PS) $)_{c}$-condition for each $c \in \mathbb{R}$.

Theorem 2.8 ([4, Theorem 4.7]) Let $X$ be a Banach space and let $\Phi \in C^{1}(X, \mathbb{R})$. Assume that $X$ splits into a direct sum of closed subspace $X=X^{-} \oplus X^{+}$with

$$
\operatorname{dim} X^{-}<\infty \text { and } \sup _{S_{R}^{-}} \Phi<\inf _{X^{+}} \Phi
$$

where $S_{R}^{-}=\left\{u \in X^{-}:\|u\|=R\right\}$. Let

$$
\begin{aligned}
& B_{R}^{-}=\left\{u \in X^{-}:\|u\| \leq R\right\}, \\
& M=\left\{h \in C\left(B_{R}^{-}, X\right): h(s)=s \text { if } s \in S_{R}^{-}\right\}
\end{aligned}
$$

and

$$
c=\inf _{h \in M} \max _{s \in B_{R}^{-}} \Phi(h(s)) .
$$

Then, if $\Phi$ satisfies the $(\mathrm{PS})_{c}$-condition, $c$ is a critical value of $\Phi$.

As shown in [34], a deformation lemma can be proved with the weaker condition (C) replacing the usual P.S. condition, and it turns out that the saddle point theorem (Theorem 2.7) holds true under condition (C).

\section{Variational setting}

In this section, in order to apply the critical point theory, we make a variational structure. From this variational structure, we can reduce the problem of finding solutions of problem (1.1) to the one of seeking the critical points of a corresponding functional.

Consider the functional $\varphi: W_{\Delta, T}^{1, p}\left([0, T]_{\mathbb{T}}, \mathbb{R}^{N}\right) \rightarrow \mathbb{R}$ defined by

$$
\varphi(u)=\frac{1}{p} \int_{[0, T)_{\mathbb{T}}}\left|u^{\Delta}(t)\right|^{p} \Delta t+\int_{[0, T)_{\mathbb{T}}} F\left(\sigma(t), u^{\sigma}(t)\right) \Delta t .
$$

We have the following facts.

Theorem 3.1 The functional $\varphi$ is continuously differentiable on $W_{\Delta, T}^{1, p}\left([0, T]_{\mathbb{T}}, \mathbb{R}^{N}\right)$ and

$$
\left\langle\varphi^{\prime}(u), v\right\rangle=\int_{[0, T)_{\mathbb{T}}}\left|u^{\Delta}\right|^{p-2}\left(u^{\Delta}(t), v^{\Delta}(t)\right) \Delta t+\int_{[0, T)_{\mathbb{T}}}\left(\nabla F\left(\sigma(t), u^{\sigma}(t)\right), v^{\sigma}(t)\right) \Delta t
$$

for all $v \in W_{\Delta, T}^{1, p}\left([0, T]_{\mathbb{T}}, \mathbb{R}^{N}\right)$.

Proof Let $L(t, x, y)=\frac{1}{p}|y|^{p}+F(t, x)$ for all $x, y \in \mathbb{R}^{N}$ and $t \in[0, T]_{\mathbb{T}}$. Then, by condition (A), $L(t, x, y)$ satisfies all the assumptions of Theorem 2.6. Hence, by Theorem 2.6, we know that the functional $\varphi$ is continuously differentiable on $W_{\Delta, T}^{1, p}\left([0, T]_{\mathbb{T}}, \mathbb{R}^{N}\right)$ and

$$
\left\langle\varphi^{\prime}(u), v\right\rangle=\int_{[0, T)_{\mathbb{T}}}\left|u^{\Delta}\right|^{p-2}\left(u^{\Delta}(t), v^{\Delta}(t)\right) \Delta t+\int_{[0, T)_{\mathbb{T}}}\left(\nabla F\left(\sigma(t), u^{\sigma}(t)\right), v^{\sigma}(t)\right) \Delta t
$$

for all $v \in W_{\Delta, T}^{1, p}\left([0, T]_{\mathbb{T}}, \mathbb{R}^{N}\right)$. 
Theorem 3.2 If $u \in W_{\Delta, T}^{1, p}\left([0, T]_{\mathbb{T}}, \mathbb{R}^{N}\right)$ is a critical point of $\varphi$ in $W_{\Delta, T}^{1, p}\left([0, T]_{\mathbb{T}}, \mathbb{R}^{N}\right)$, i.e., $\varphi^{\prime}(u)=0$, then $u$ is solution of problem (1.1).

Proof Since $\varphi^{\prime}(u)=0$, it follows from Theorem 3.1 that

$$
\int_{[0, T)_{\mathbb{T}}}\left|u^{\Delta}\right|^{p-2}\left(u^{\Delta}(t), v^{\Delta}(t)\right) \Delta t+\int_{[0, T)_{\mathbb{T}}}\left(\nabla F\left(\sigma(t), u^{\sigma}(t)\right), v^{\sigma}(t)\right) \Delta t=0
$$

for all $v \in W_{\Delta, T}^{1, p}\left([0, T]_{\mathbb{T}}, \mathbb{R}^{N}\right)$, that is,

$$
\int_{[0, T)_{\mathbb{T}}}\left|u^{\Delta}\right|^{p-2}\left(u^{\Delta}(t), v^{\Delta}(t)\right) \Delta t=-\int_{[0, T)_{\mathbb{T}}}\left(\nabla F\left(\sigma(t), u^{\sigma}(t)\right), v^{\sigma}(t)\right) \Delta t
$$

for all $v \in W_{\Delta, T}^{1, p}\left([0, T]_{\mathbb{T}}, \mathbb{R}^{N}\right)$. From condition (A) and Definition 2.10, we have that $\left|u^{\Delta}\right|^{p-2} u^{\Delta} \in W_{\Delta, T}^{1, p}\left([0, T]_{\mathbb{T}}, \mathbb{R}^{N}\right)$. By Theorem 2.2 and (2.3), there exists a unique function $x \in V_{\Delta, T}^{1, p}\left([0, T]_{\mathbb{T}}, \mathbb{R}^{N}\right)$ such that

$$
x=u,\left(\left|x^{\Delta}(t)\right|^{p-2} x^{\Delta}(t)\right)^{\Delta}=\nabla F\left(\sigma(t), u^{\sigma}(t)\right), \quad \Delta \text {-a.e. on }[0, T]_{\mathbb{T}}^{\kappa}
$$

and

$$
\int_{[0, T)_{\mathbb{T}}} \nabla F\left(\sigma(t), u^{\sigma}(t)\right) \Delta t=0
$$

Combining (3.3) and (3.4), we obtain

$$
x(0)-x(T)=0, \quad x^{\Delta}(0)-x^{\Delta}(T)=0 .
$$

We identify $u \in W_{\Delta, T}^{1, p}\left([0, T]_{\mathbb{T}}, \mathbb{R}^{N}\right)$ with its absolutely continuous representative $x \in$ $V_{\Delta, T}^{1,2}\left([0, T]_{\mathbb{T}}, \mathbb{R}^{N}\right)$ for which (3.3) holds. By Theorem 2.7, $u$ is a $T$-periodic solution of problem (1.1).

\section{Main results}

For $u \in W_{\Delta, T}^{1, p}\left([0, T]_{\mathbb{T}}, \mathbb{R}^{N}\right)$, let $\bar{u}=\frac{1}{T} \int_{[0, T)_{\mathbb{T}}} u(t) \Delta t$ and $\widetilde{u}(t)=u(t)-\bar{u}$. Set $\widetilde{W}_{\Delta, T}^{1, p}\left([0, T]_{\mathbb{T}}\right.$, $\left.\mathbb{R}^{N}\right)=\left\{u \in W_{\Delta, T}^{1, p}\left([0, T]_{\mathbb{T}}, \mathbb{R}^{N}\right): \int_{[0, T)_{\mathbb{T}}} u(t) \Delta t=0\right\}$, then $W_{\Delta, T}^{1, p}\left([0, T]_{\mathbb{T}}, \mathbb{R}^{N}\right)=\widetilde{W}_{\Delta, T}^{1, p}\left([0, T]_{\mathbb{T}}\right.$, $\left.\mathbb{R}^{N}\right) \oplus \mathbb{R}^{N}$ and we have the following lemma.

Theorem 4.1 In the Sobolev space $W_{\Delta, T}^{1, p}\left([0, T]_{\mathbb{T}}, \mathbb{R}^{N}\right)$, for $u \in W_{\Delta, T}^{1, p}\left([0, T]_{\mathbb{T}}, \mathbb{R}^{N}\right),\|u\| \rightarrow \infty$ if and only if

$$
\left(|\bar{u}|^{p}+\int_{[0, T)}\left|u^{\Delta}(t)\right|^{p} \Delta t\right)^{\frac{1}{p}} \rightarrow \infty
$$

Proof On the one hand, from Theorem 2.4, we get

$$
\begin{aligned}
\int_{[0, T)_{\mathbb{T}}}|u(t)|^{p} \Delta t & =\int_{[0, T)_{\mathbb{T}}}|\bar{u}+\widetilde{u}(t)|^{p} \Delta t \\
& \leq 2^{p} \int_{[0, T)_{\mathbb{T}}}\left(|\bar{u}|^{p}+|\widetilde{u}(t)|^{p}\right) \Delta t
\end{aligned}
$$




$$
\begin{aligned}
& \leq 2^{p} T|\bar{u}|^{p}+2^{p} T\|\widetilde{u}(t)\|_{\infty}^{p} \\
& \leq 2^{p} T|\bar{u}|^{p}+2^{p} T K^{p} \int_{[0, T)_{\mathbb{T}}}\left|u^{\Delta}(t)\right|^{p} \Delta t .
\end{aligned}
$$

Therefore, one has

$$
\begin{aligned}
\|u\| & =\left(\int_{[0, T)_{\mathbb{T}}}|u(t)|^{p} \Delta t+\int_{[0, T)_{\mathbb{T}}}\left|u^{\Delta}(t)\right|^{p} \Delta t\right)^{\frac{1}{p}} \\
& \leq\left(2^{p} T|\bar{u}|^{p}+2^{p} T K^{p} \int_{[0, T)_{\mathbb{T}}}\left|u^{\Delta}(t)\right|^{p} \Delta t+\int_{[0, T)_{\mathbb{T}}}\left|u^{\Delta}(t)\right|^{p} \Delta t\right)^{\frac{1}{p}} \\
& \leq\left(2^{p} T+2^{p} T K^{p}+1\right)^{\frac{1}{p}}\left(|\bar{u}|^{p}+\int_{[0, T)_{\mathbb{T}}}\left|u^{\Delta}(t)\right|^{p} \Delta t\right)^{\frac{1}{p}} .
\end{aligned}
$$

On the other hand, by the Hölder inequality, we have

$$
|\bar{u}|=\left|\frac{1}{T} \int_{[0, T)_{\mathbb{T}}} u(t) \Delta t\right| \leq T^{-\frac{1}{p}}\left(\int_{[0, T)_{\mathbb{T}}}|u(t)|^{p} \Delta t\right)^{\frac{1}{p}} .
$$

Hence, we get

$$
\begin{aligned}
\left(|\bar{u}|^{p}+\int_{[0, T)_{\mathbb{T}}}\left|u^{\Delta}(t)\right|^{p} \Delta t\right)^{\frac{1}{p}} & \leq\left(T^{-1}\left(\int_{[0, T)_{\mathbb{T}}}|u(t)|^{p} \Delta t\right)+\int_{[0, T)_{\mathbb{T}}}\left|u^{\Delta}(t)\right|^{p} \Delta t\right)^{\frac{1}{p}} \\
& \leq\left(T^{-1}+1\right)^{\frac{1}{p}}\|u\| .
\end{aligned}
$$

It follows from (4.1) and (4.2) that $\|u\| \rightarrow \infty$ if and only if

$$
\left(|\bar{u}|^{p}+\int_{[0, T)_{\mathbb{T}}}\left|u^{\Delta}(t)\right|^{p} \Delta t\right)^{\frac{1}{p}} \rightarrow \infty .
$$

Theorem 4.2 Suppose that (A) and the following conditions are satisfied.

$\left(\mathrm{F}_{1}\right)$ There exist $0<\mu<p, M>0$ such that

$$
(\nabla F(t, x), x) \geq \mu F(t, x)
$$

for all $|x|>M$ and $\Delta$-a.e. $t \in[0, T]_{\mathbb{T}}$.

$\left(\mathrm{F}_{2}\right)$ There exists $g^{\sigma} \in L_{\Delta}^{1}\left([0, T]_{\mathbb{T}}, \mathbb{R}\right)$ such that

$$
F(t, x) \leq g(t)
$$

for all $x \in \mathbb{R}^{N}$ and $\Delta$-a.e. $t \in[0, T]_{\mathbb{T}}$.

$\left(\mathrm{F}_{3}\right)$ There exists a subset $D$ of $[0, T]_{\mathbb{T}}$ with $\mu_{\Delta}(D)>0$ such that

$$
F(t, x) \rightarrow-\infty \quad \text { as }|x| \rightarrow \infty
$$

for $\Delta$-a.e. $t \in D$.

Then problem (1.1) has at least one T-periodic solution. 
Proof We will use the saddle point theorem only to prove that

(i) $\varphi$ satisfies (C)-condition.

(ii) $\varphi(u) \rightarrow-\infty$ as $u \in \mathbb{R}^{N},\|u\| \rightarrow \infty$.

(iii) $\varphi(u) \rightarrow+\infty$ as $u \in \widetilde{W}_{\Delta, T}^{1, p}\left([0, T]_{\mathbb{T}}, \mathbb{R}^{N}\right),\|u\| \rightarrow \infty$.

Firstly, we prove (i).

Set $\left\{u_{n}\right\} \subset W_{\Delta, T}^{1, p}\left([0, T]_{\mathbb{T}}, \mathbb{R}^{N}\right)$ is a $(C)$-sequence, that is, $\varphi\left(u_{n}\right)$ is bounded and $(1+$ $\left.\left\|u_{n}\right\|\right) \varphi^{\prime}\left(u_{n}\right) \rightarrow 0$ as $n \rightarrow \infty$. Then there exists a positive constant $C_{1}$ such that

$$
\left|\varphi\left(u_{n}\right)\right| \leq C_{1}, \quad\left(1+\left\|u_{n}\right\|\right)\left\|\varphi^{\prime}\left(u_{n}\right)\right\| \leq C_{1}
$$

for all $n \in \mathbb{N}$. Using (A), ( $\left.\mathrm{F}_{1}\right),(3.1)$ and (3.2), one has

$$
\begin{aligned}
C_{1}+p C_{1} \geq & \left(1+\left\|u_{n}\right\|\right)\left\|\varphi^{\prime}\left(u_{n}\right)\right\|-p \varphi\left(u_{n}\right) \\
& \geq\left\langle\varphi^{\prime}\left(u_{n}\right), u_{n}\right\rangle-p \varphi\left(u_{n}\right) \\
& =\int_{[0, T)_{\mathbb{T}}}\left[\left(\nabla F\left(\sigma(t), u_{n}^{\sigma}(t)\right), u_{n}^{\sigma}(t)\right)-p F\left(\sigma(t), u_{n}^{\sigma}(t)\right)\right] \Delta t \\
& \geq(\mu-p) \int_{[0, T)_{\mathbb{T}}} F\left(\sigma(t), u_{n}^{\sigma}(t)\right) \Delta t-(p+M) \max _{|x| \leq M} a(|x|) \int_{[0, T)_{\mathbb{T}}} b^{\sigma}(t) \Delta t
\end{aligned}
$$

for all $n \in \mathbb{N}$. This implies that there exists a constant $C_{2}$ such that

$$
\int_{[0, T)_{\mathbb{T}}} F\left(\sigma(t), u_{n}^{\sigma}(t)\right) \Delta t \geq C_{2}
$$

for all $n \in \mathbb{N}$. From (3.1), (4.3) and (4.4), we have

$$
\begin{aligned}
C_{1} & \geq \varphi\left(u_{n}\right) \\
& \geq \frac{1}{p} \int_{[0, T)_{\mathbb{T}}}\left|u_{n}^{\Delta}(t)\right|^{p} \Delta t+C_{2}
\end{aligned}
$$

for all $n \in \mathbb{N}$. This implies that there exists a constant $C_{3}$ such that

$$
\int_{[0, T)_{\mathbb{T}}}\left|u_{n}^{\Delta}(t)\right|^{p} \Delta t \leq C_{3}
$$

for all $n \in \mathbb{N}$. It follows from Theorem 2.4 and (4.5) that there exists a constant $C_{4}$ such that

$$
\left\|\widetilde{u}_{n}\right\|_{\infty} \leq C_{4}
$$

for all $n \in \mathbb{N}$

Now, we will prove that the sequence $\left\{\bar{u}_{n}\right\}$ is bounded. Otherwise, we may assume, without loss of generality, that $\left|\bar{u}_{n}\right| \rightarrow \infty$ as $n \rightarrow \infty$. Set $v_{n}=\frac{u_{n}}{\left\|u_{n}\right\|}=\frac{\bar{u}_{n}}{\left\|u_{n}\right\|}+\frac{\widetilde{u}_{n}}{\left\|u_{n}\right\|}=\bar{v}_{n}+\widetilde{v}_{n}$, then $\left\{v_{n}\right\}$ is bounded in $W_{\Delta, T}^{1, p}\left([0, T]_{\mathbb{T}}, \mathbb{R}^{N}\right)$. By Remark 2.1, there is a subsequence of $\left\{v_{n}\right\}$ (for simplicity denoted again by $\left.\left\{v_{n}\right\}\right)$ such that

$$
\begin{array}{ll}
v_{n} \rightarrow v & \text { in } W_{\Delta, T}^{1, p}\left([0, T]_{\mathbb{T}}, \mathbb{R}^{N}\right), \\
v_{n} \rightarrow v & \text { in } C\left([0, T]_{\mathbb{T}}, \mathbb{R}^{N}\right) .
\end{array}
$$


Combining with (4.6), $\left\{\tilde{u}_{n}\right\}$ is bounded in $C\left([0, T]_{\mathbb{T}}, \mathbb{R}^{N}\right)$, thereby, $v \in \mathbb{R}^{N}, v \neq 0$. So, $\left|u_{n}(t)\right| \rightarrow \infty$ as $n \rightarrow \infty$ for all $t \in[0, T]_{\mathbb{T}}$. By $\left(\mathrm{F}_{3}\right)$, we have

$$
\begin{aligned}
& \limsup _{n \rightarrow \infty} \int_{[0, T)_{\mathbb{T}}} F\left(\sigma(t), u_{n}^{\sigma}(t)\right) \Delta t \\
& \quad \leq \limsup _{n \rightarrow \infty} \int_{D} F\left(\sigma(t), u_{n}^{\sigma}(t)\right) \Delta t+\int_{[0, T)_{\mathbb{T}}}\left|g^{\sigma}(t)\right| \Delta t \\
& \quad \rightarrow-\infty,
\end{aligned}
$$

which contradicts (4.4).

Then, by Theorem 4.1, $\left\{u_{n}\right\}$ is bounded in $W_{\Delta, T}^{1, p}\left([0, T]_{\mathbb{T}}, \mathbb{R}^{N}\right)$. Again, from Remark 2.1, there is a subsequence of $\left\{u_{n}\right\}$ (for simplicity denoted again by $\left\{u_{n}\right\}$ ) such that

$$
\begin{array}{ll}
u_{n} \rightarrow u & \text { in } W_{\Delta, T}^{1, p}\left([0, T]_{\mathbb{T}}, \mathbb{R}^{N}\right), \\
u_{n} \rightarrow u & \text { in } C\left([0, T]_{\mathbb{T}}, \mathbb{R}^{N}\right) .
\end{array}
$$

By (4.8), $\left\{u_{n}\right\}$ is bounded in $C\left([0, T]_{\mathbb{T}}, \mathbb{R}^{N}\right)$, then by assumption (A), there exists a positive constant $C_{5}$ such that

$$
\begin{aligned}
\left|\int_{[0, T)_{\mathbb{T}}}\left(\nabla F\left(\sigma(t), u_{n}^{\sigma}(t)\right), u^{\sigma}(t)-u_{n}^{\sigma}(t)\right) \Delta t\right| & \leq \int_{[0, T)_{\mathbb{T}}} \mid \nabla F\left(\sigma(t), u_{n}^{\sigma}(t)|| u^{\sigma}(t)-u_{n}^{\sigma}(t) \mid \Delta t\right. \\
& \leq C_{5} \int_{[0, T)_{\mathbb{T}}} b^{\sigma}(t)\left|u^{\sigma}(t)-u_{n}^{\sigma}(t)\right| \Delta t \\
& \leq C_{5}\left\|u-u_{n}\right\|_{\infty} \int_{[0, T)_{\mathbb{T}}} b^{\sigma}(t) \Delta t .
\end{aligned}
$$

Combining with (4.8), we obtain

$$
\int_{[0, T)_{\mathbb{T}}}\left(\nabla F\left(\sigma(t), u_{n}^{\sigma}(t)\right), u^{\sigma}(t)-u_{n}^{\sigma}(t)\right) \Delta t \rightarrow 0 \quad \text { as } n \rightarrow \infty
$$

Note that

$$
\begin{aligned}
\left\langle\varphi^{\prime}\left(u_{n}\right), u-u_{n}\right\rangle= & \int_{[0, T)_{\mathbb{T}}}\left|u_{n}^{\Delta}\right|^{p-2}\left(u_{n}^{\Delta}(t), u^{\Delta}(t)-u_{n}^{\Delta}(t)\right) \Delta t \\
& +\int_{[0, T)_{\mathbb{T}}}\left[\nabla F\left(\sigma(t), u_{n}^{\sigma}(t)\right), u^{\sigma}(t)-u_{n}^{\sigma}(t)\right] \Delta t
\end{aligned}
$$

and

$$
\left\langle\varphi^{\prime}\left(u_{n}\right), u-u_{n}\right\rangle \rightarrow 0 \quad \text { as } n \rightarrow \infty .
$$

Hence, we have

$$
\int_{[0, T)_{\mathbb{T}}}\left|u_{n}^{\Delta}\right|^{p-2}\left(u_{n}^{\Delta}(t), u^{\Delta}(t)-u_{n}^{\Delta}(t)\right) \Delta t \rightarrow 0 \quad \text { as } n \rightarrow \infty .
$$


Furthermore, it is easy to derive from (4.8) that

$$
\int_{[0, T)_{\mathbb{T}}}\left|u_{n}(t)\right|^{p-2}\left(u_{n}(t), u(t)-u_{n}(t)\right) \Delta t \rightarrow 0 \quad \text { as } n \rightarrow \infty .
$$

Let

$$
\phi(u)=\frac{1}{p}\|u\|^{p}=\frac{1}{p}\left(\int_{[0, T)_{\mathbb{T}}}|u(t)|^{p} \Delta t+\int_{[0, T)_{\mathbb{T}}}\left|u^{\Delta}(t)\right|^{p} \Delta t\right) .
$$

Then one has

$$
\begin{aligned}
& \left\langle\phi^{\prime}\left(u_{n}\right), u-u_{n}\right\rangle \\
& \quad=\int_{[0, T)_{\mathbb{T}}}\left|u_{n}^{\Delta}\right|^{p-2}\left(u_{n}^{\Delta}(t), u^{\Delta}(t)-u_{n}^{\Delta}(t)\right) \Delta t+\int_{[0, T)_{\mathbb{T}}}\left|u_{n}(t)\right|^{p-2}\left(u_{n}(t), u(t)-u_{n}(t)\right) \Delta t \\
& \quad \rightarrow 0
\end{aligned}
$$

as $n \rightarrow \infty$. By Hölder inequality, we get

$$
0 \leq\left(\left\|u_{n}\right\|-\|u\|\right)\left(\left\|u_{n}\right\|^{p-1}-\|u\|^{p-1}\right) \leq\left\langle\phi^{\prime}\left(u_{n}\right)-\phi^{\prime}(u), u_{n}-u\right\rangle .
$$

This combined with (4.9) yields $\left\|u_{n}\right\| \rightarrow\|u\|$. It follows from (4.7) and uniform convexity of $W_{\Delta, T}^{1, p}\left([0, T]_{\mathbb{T}}, \mathbb{R}^{N}\right)$ that $u_{n} \rightarrow u$ in $W_{\Delta, T}^{1, p}\left([0, T]_{\mathbb{T}}, \mathbb{R}^{N}\right)$.

Secondly, we prove (ii).

$\forall u \in \mathbb{R}^{N}$, by $(3.1),\left(\mathrm{F}_{2}\right)$ and $\left(\mathrm{F}_{3}\right)$, we obtain

$$
\begin{aligned}
\varphi(u) & =\int_{[0, T)_{\mathbb{T}}} F\left(\sigma(t), u^{\sigma}(t)\right) \Delta t \\
& \leq \int_{D} F\left(\sigma(t), u^{\sigma}(t)\right) \Delta t+\int_{[0, T)_{\mathbb{T}} \backslash D} g^{\sigma}(t) \Delta t \\
& \leq \int_{D} F\left(\sigma(t), u^{\sigma}(t)\right) \Delta t+\int_{[0, T)_{\mathbb{T}}}\left|g^{\sigma}(t)\right| \Delta t \\
& \rightarrow-\infty
\end{aligned}
$$

as $n \rightarrow \infty$. This implies that (ii) holds.

Thirdly, we prove (iii).

For $s \in \mathbb{R},|x| \geq M$ and $\Delta$-a.e. $t \in[0, T]_{\mathbb{T}}$, let

$$
G(s)=F(t, s x), \quad H(s)=G^{\prime}(s)-\frac{\mu}{s} G(s) .
$$

Using $\left(\mathrm{F}_{1}\right)$, when $s \geq \frac{M}{|x|}$,

$$
H(s)=\frac{1}{s}((\nabla F(t, s x), s x)-\mu F(t, s x)) \geq 0
$$

holds. Furthermore, by (4.10), G(s) satisfies

$$
G^{\prime}(s)=H(s)+\frac{\mu}{s} G(s) .
$$


Hence, when $s \geq \frac{M}{|x|}$,

$$
F(t, s x)=s^{\mu}\left(F(t, x)+\int_{1}^{s} \tau^{-\mu} H(\tau) \mathrm{d} \tau\right) .
$$

Again, by virtue of assumption (A) and (4.11), for $|x| \geq M$ and $\Delta$-a.e. $t \in[0, T]_{\mathbb{T}}$, we assert

$$
\left(\frac{M}{|x|}\right)^{\mu} F(t, x) \geq F\left(t, x \frac{M}{|x|}\right) \geq-a_{0} b(t),
$$

where $a_{0}=\max _{|x| \leq M} a(|x|)$. This implies that

$$
F(t, x) \geq-a_{0} b(t)\left(\left(\frac{|x|}{M}\right)^{\mu}+1\right)
$$

for all $x \in \mathbb{R}^{N}$ and $\Delta$-a.e. $t \in[0, T]_{\mathbb{T}}$. Moreover, by Theorem 2.4 and (3.1), we get

$$
\begin{aligned}
\varphi(u) \geq & \frac{1}{p} \int_{[0, T)_{\mathbb{T}}}\left|u^{\Delta}(t)\right|^{p} \Delta t-\left(\frac{a_{0}}{M^{\mu}}\right)\|u\|_{\infty}^{\mu} \int_{[0, T)_{\mathbb{T}}} b^{\sigma}(t) \Delta t-a_{0} \int_{[0, T)_{\mathbb{T}}} b^{\sigma}(t) \Delta t \\
= & \frac{1}{p}\|u\|^{p}-\frac{1}{p} \int_{[0, T)_{\mathbb{T}}}|u(t)|^{p} \Delta t \\
& -\left(\frac{a_{0}}{M^{\mu}}\right)\|u\|_{\infty}^{\mu} \int_{[0, T)_{\mathbb{T}}} b^{\sigma}(t) \Delta t-a_{0} \int_{[0, T)_{\mathbb{T}}} b^{\sigma}(t) \Delta t \\
\geq & \frac{1}{p}\|u\|^{p}-\frac{1}{p} T\|u\|_{\infty}^{p}-\left(\frac{a_{0}}{M^{\mu}}\right)\|u\|_{\infty}^{\mu} \int_{[0, T)_{\mathbb{T}}} b^{\sigma}(t) \Delta t-a_{0} \int_{[0, T)_{\mathbb{T}}} b^{\sigma}(t) \Delta t \\
\geq & \frac{1}{p}\|u\|^{p}-\frac{T K^{p}}{p}\|u\|^{p}-\left(\frac{a_{0} K^{p}}{M^{\mu}}\right)\|u\|^{\mu} \int_{[0, T)_{\mathbb{T}}} b^{\sigma}(t) \Delta t-a_{0} \int_{[0, T)_{\mathbb{T}}} b^{\sigma}(t) \Delta t
\end{aligned}
$$

for all $u \in \widetilde{W}_{\Delta, T}^{1, p}\left([0, T]_{\mathbb{T}}, \mathbb{R}^{N}\right)$. This implies that (ii) holds.

It follows from the saddle point theorem and Theorem 3.2 that Theorem 4.2 holds.

Example 4.1 Let $\mathbb{T}=\bigcup_{k=0}^{\infty}[2 k, 2 k+1], T=2, N=4$. Consider the 4-Laplacian system on the time scale $\mathbb{T}$

$$
\left(\left|u^{\Delta}(t)\right|^{2} u^{\Delta}(t)\right)^{\Delta}=\nabla F(\sigma(t), u(\sigma(t))), \quad \Delta \text {-a.e. } t \in \mathbb{T}^{\kappa}
$$

where $F(t, x)=-|x|^{2}-|((1,1,2,3), x)|$.

Since $F(t, x)=-|x|^{2}-|((1,2,2,3), x)|, \nabla F(t, x)=-2 x-\frac{((1,1,2,3), x)}{|((1,1,2,3), x)|}(1,1,2,3)$, all the conditions of Theorem 4.2 hold with $p=4, \mu=3, M=1, g(t) \equiv 0$. According to Theorem 4.2, problem (4.12) has at least one 2-periodic solution. Moreover, 0 is not the solution of problem (4.12). Thus, problem (4.12) has at least one nontrivial 2-periodic solution.

From Theorem 4.2, we have the following corollary.

Corollary 4.1 Suppose that $(\mathrm{A}),\left(\mathrm{F}_{1}\right)$ and the following condition are satisfied.

$\left(\mathrm{F}_{4}\right) \quad F(t, x) \rightarrow-\infty$ as $|x| \rightarrow \infty$ uniformly for $\Delta$-a.e. $t \in[0, T]_{\mathbb{T}}$.

Then problem (1.1) has at least one T-periodic solution. 
Theorem 4.3 Suppose that (A), $\left(\mathrm{F}_{1}\right)$ and the following conditions are satisfied.

$\left(\mathrm{F}_{5}\right) \int_{[0, T)_{\mathbb{T}}} F(\sigma(t), x) \Delta t \rightarrow-\infty$ as $|x| \rightarrow \infty$.

$\left(\mathrm{F}_{6}\right)$ Assume that $-F(t, \cdot)$ is $(\beta, \gamma)$-subconvex with $\gamma>0 \Delta$-a.e. $t \in[0, T]_{\mathbb{T}}$, that is,

$$
\begin{array}{r}
F(t, \beta(x+y)) \geq \gamma(F(t, x)+F(t, y)) \\
\text { for all } x, y \in \mathbb{R}^{N} \text { and } \Delta \text {-a.e. } t \in[0, T]_{\mathbb{T}} .
\end{array}
$$

Then problem (1.1) has at least one T-periodic solution.

Proof It follows directly from $\left(\mathrm{F}_{5}\right)$ that

$$
\varphi(u) \rightarrow-\infty \quad \text { as } u \in \mathbb{R}^{N},\|u\| \rightarrow \infty .
$$

Similar to the proof of Theorem 4.2, we can prove

$$
\varphi(u) \rightarrow+\infty \quad \text { as } u \in \widetilde{W}_{\Delta, T}^{1, p}\left([0, T]_{\mathbb{T}}, \mathbb{R}^{N}\right),\|u\| \rightarrow \infty .
$$

Next, we will prove that $\varphi$ satisfies (C)-condition. Let $\left\{u_{n}\right\} \subset W_{\Delta, T}^{1, p}\left([0, T]_{\mathbb{T}}, \mathbb{R}^{N}\right)$ be a $(\mathrm{C})$ sequence, that is, $\varphi\left(u_{n}\right)$ is bounded and $\left(1+\left\|u_{n}\right\|\right) \varphi^{\prime}\left(u_{n}\right) \rightarrow 0$ as $n \rightarrow \infty$. In a way similar to (4.4), (4.5) and (4.6) in the proof of (i) in Theorem 4.2, there exist constants $C_{6}, C_{7}, C_{8}$ such that

$$
\begin{aligned}
& \int_{[0, T)_{\mathbb{T}}} F\left(\sigma(t), u_{n}^{\sigma}(t)\right) \Delta t \geq C_{6}, \\
& \int_{[0, T)_{\mathbb{T}}}\left|u_{n}^{\Delta}(t)\right|^{p} \Delta t \leq C_{7}
\end{aligned}
$$

and

$$
\left\|\tilde{u}_{n}\right\|_{\infty} \leq C_{8}
$$

for all $n \in \mathbb{N}$. Using $\left(\mathrm{F}_{6}\right)$, we obtain

$$
\begin{aligned}
C_{6} & \leq \int_{[0, T)_{\mathbb{T}}} F\left(\sigma(t), u_{n}^{\sigma}(t)\right) \Delta t \\
& \leq \frac{1}{\gamma} \int_{[0, T)_{\mathbb{T}}} F\left(\sigma(t), \beta \bar{u}_{n}\right) \Delta t-\int_{[0, T)_{\mathbb{T}}} F\left(\sigma(t),-\widetilde{u}_{n}^{\sigma}(t)\right) \Delta t \\
& \leq \frac{1}{\gamma} \int_{[0, T)_{\mathbb{T}}} F\left(\sigma(t), \beta \bar{u}_{n}\right) \Delta t-\max _{|x| \leq C_{8}} a(|x|) \int_{[0, T)_{\mathbb{T}}} b^{\sigma}(t) \Delta t
\end{aligned}
$$

for all $n \in \mathbb{N}$, which implies that $\left\{\bar{u}_{n}\right\}$ is bounded. Combining with (4.13), we assert that $\left\{u_{n}\right\}$ is bounded. Using the same method as that of the proof of (i) in Theorem 4.2, we can prove that $\left\{u_{n}\right\}$ has a convergent subsequence, thus, $\varphi$ satisfies condition (C).

By the saddle point theorem and Theorem 3.2, Theorem 4.3 holds. 
Example 4.2 Let $\mathbb{T}=\mathbb{Z}, T=20, N=3$. Consider the 5 -Laplacian system on the time scale $\mathbb{T}$

$$
\Delta\left(|\Delta u(t)|^{3} \Delta u(t)\right)=\nabla F(t+1, u(t+1)), \quad \Delta \text {-a.e. } t \in \mathbb{Z},
$$

where $F(t, x)=-|x|^{3}-|((1,1,1), x)|$.

Since $F(t, x)=-|x|^{3}-|((1,1,1), x)|, \nabla F(t, x)=-3|x| x-\frac{((1,1,1), x)}{|((1,1,1), x)|}(1,1,1)$, all the conditions of Theorem 4.3 hold $p=5, \mu=4, M=2$. According to Theorem 4.3, problem (4.14) has at least one 20-periodic solution. Moreover, 0 is not the solution of problem (4.14). Thus, problem (4.14) has at least one nontrivial 20-periodic solution.

\section{Competing interests}

The authors declare that they have no competing interests.

\section{Authors' contributions}

All authors contributed equally to the manuscript and typed, read and approved the final manuscript.

\section{Acknowledgements}

This work was supported by the National Natural Sciences Foundation of People's Republic of China under Grant 11361072 and the Natural Sciences Foundation of Yunnan Province under Grant 2012 FB111.

Received: 9 July 2013 Accepted: 25 September 2013 Published: 07 Nov 2013

\section{References}

1. Ma, SW, Zhang, YX: Existence of infinitely many periodic solutions for ordinary $p$-Laplacian systems. J. Math. Anal. Appl. 351, 469-479 (2009)

2. Li, C, Ou, ZQ, Tang, CL: Three periodic solutions for p-Hamiltonian systems. Nonlinear Anal. 74, 1596-1606 (2011)

3. Zhou, JW, Li, YK: Sobolev's spaces on time scales and its applications to a class of second order Hamiltonian systems on time scales. Nonlinear Anal. 73, 1375-1388 (2010)

4. Mawhin, J, Willem, M: Critical Point Theory and Hamiltonian Systems. Springer, Berlin (1989)

5. Berger, MS, Schechter, M: On the solvability of semilinear gradient operator equations. Adv. Math. 25, 97-132 (1977)

6. Long, YM: Nonlinear oscillations for classical Hamiltonian systems with bi-even subquadratic potentials. Nonlinear Anal. 24, 1665-1671 (1995)

7. Rabinowitz, PH: On subharmonic solutions of Hamiltonian systems. Commun. Pure Appl. Math. 33, 609-633 (1980)

8. Tang, CL: Periodic solutions for nonautonomous second systems with sublinear nonlinearity. Proc. Am. Math. Soc. $126,3263-3270(1998)$

9. Xue, YF, Tang, CL: Multiple periodic solutions for superquadratic second-order discrete Hamiltonian systems. Appl. Math. Comput. 196, 494-500 (2008)

10. Yu, JS, Deng, XQ, Guo, ZM: Periodic solutions of a discrete Hamiltonian system with a change of sign in the potential. J. Math. Anal. Appl. 324, 1140-1151 (2006)

11. Aulbach, B, Hilger, S: Linear dynamic processes with inhomogeneous time scale. In: Nonlinear Dynamics and Quantum Dynamical Systems. Mathematical Research, vol. 59, pp. 9-20. Akademie Verlag, Berlin (1990)

12. Erbe, L, Hilger, S: Sturmanian theory on measure chains. Differ. Equ. Dyn. Syst. 1(3), 223-244 (1993)

13. Lakshmikantham, V, Sivasundaram, S, Kaymakcalan, B: Dynamic Systems on Measure Chains. Kluwer Academic, Dordrecht (1996)

14. Agarwal, RP, Bohner, M: Basic calculus on time scales and some of its applications. Results Math. 35(1-2), 3-22 (1999)

15. Bohner, M, Peterson, A: Dynamic Equations on Time Scales: An Introduction with Applications. Birkhäuser, Boston (2001)

16. Bohner, M, Peterson, A: Advances in Dynamic Equations on Time Scales. Birkhäuser, Boston (2003)

17. Zhang, HT, Li, YK: Existence of positive periodic solutions for functional differential equations with impulse effects on time scales. Commun. Nonlinear Sci. Numer. Simul. 14, 19-26 (2009)

18. Li, YK, Zhao, LL, Zhang, TW: Global exponential stability and existence of periodic solution of impulsive Cohen-Grossberg neural networks with distributed delays on time scales. Neural Process. Lett. 33, 61-81 (2011)

19. Hao, ZC, Xiao, TJ, Liang, J: Existence of positive solutions for singular boundary value problem on time scales. J. Math. Anal. Appl. 325, 517-528 (2007)

20. Jiang, LQ, Zhou, Z: Existence of weak solutions of two-point boundary value problems for second-order dynamic equations on time scales. Nonlinear Anal. 69, 1376-1388 (2008)

21. Agarwal, RP, Bohner, M, Řehák, P: Half-linear dynamic equations. In: Nonlinear Analysis and Applications: To V. Lakshmikantham on His 80th Birthday, vol. 1, pp. 1-57. Kluwer Academic, Dordrecht (2003)

22. Agarwal, RP, Bohner, M, Wong, PJY: Sturm-Liouville eigenvalue problems on time scales. Appl. Math. Comput. 99, 153-166 (1999)

23. Rynne, BP: $L^{2}$ spaces and boundary value problems on time-scales. J. Math. Anal. Appl. 328, 1217-1236 (2007)

24. Li, YK, Zhou, JW: Existence of solutions for a class of damped vibration problems on time scales. Adv. Differ. Equ. 2010 Article ID 727486 (2010) 
25. Li, YK, Wang, C: Pseudo almost periodic functions and pseudo almost periodic solutions to dynamic equations on time scales. Adv. Differ. Equ. 2012, Article ID 77 (2012)

26. Bonanno, G, Livrea, R: Multiple periodic solutions for Hamiltonian systems with not coercive potential. J. Math. Anal. Appl. 363, 627-638 (2010)

27. Tang, CL, Wu, XP: Some critical point theorems and their applications to periodic solution for second order Hamiltonian systems. J. Differ. Equ. 248, 660-692 (2010)

28. Chen, SJ, Tang, CL: Multiple periodic solutions for superquadratic first-order discrete Hamiltonian systems. Appl. Math. Comput. 208, 495-500 (2009)

29. Yu, J, Deng, X, Guo, Z: Periodic solutions of a discrete Hamiltonian system with a change of sign in the potential. J. Math. Anal. Appl. 324, 1140-1151 (2006)

30. Davidson, FA, Rynne, BP: Eigenfunction expansions in $L^{2}$ spaces for boundary value problems on time-scales. J. Math Anal. Appl. 335, 1038-1051 (2007)

31. Rynne, BP: $L^{2}$ spaces and boundary value problems on time-scales. J. Math. Anal. Appl. 328, 1217-1236 (2007)

32. Su, YH, Feng, Z: A non-autonomous Hamiltonian system on time scales. Nonlinear Anal. 75, 4126-4136 (2012)

33. Zhou, JW, Li, YK: Variational approach to a class of second order Hamiltonian systems on time scales. Acta Appl. Math. 117(1), 47-69 (2012)

34. Guseinov, GS: Integration on time scales. J. Math. Anal. Appl. 285, 107-127 (2003)

10.1186/1687-1847-2013-297

Cite this article as: Zhou and Li: Variational approach to a class of $p$-Laplacian systems on time scales. Advances in Difference Equations 2013, 2013:297

\section{Submit your manuscript to a SpringerOpen ${ }^{\circ}$ journal and benefit from:}

- Convenient online submission

- Rigorous peer review

- Immediate publication on acceptance

- Open access: articles freely available online

- High visibility within the field

- Retaining the copyright to your article 